\title{
Dietary contents, especially the vitamins are a hope in Covid-19
}

\begin{abstract}
The severe acute respiratory distress syndrome coronavirus 2 (SARS-CoV-2) breakout has caused millions of infections with thousands of deaths worldwide. Still, the number of infected cases with deaths is increasing day by day. Unfortunately, to date, we do not have a single specific remedy for it. Along with the protective measures, respiratory and/ or circulatory supports and some non-specific anti-viral drug therapies have been reported to fight against this pandemic. So far, 165 vaccine candidates have been in various stages of clinical trials, of which 26 are in human trials. Besides Oxford University's vaccine many others have already been able to garner worldwide response, it is still a long way to go. On the other hand, the discovery of vaccines is also delaying drug therapy approaches. In this case, we can focus on dietary supplements, especially vitamin-rich foods, as an alternative treatment. This writing focuses on the effects of dietary contents, especially the diets containing vitamins on the management of coronavirus disease 2019 (Covid-19).
\end{abstract}

Volume I 3 Issue 4 - 2020

\author{
Muhammad Torequl Islam \\ Department of Pharmacy, Bangabandhu Sheikh Mujibur Rahman \\ Science and Technology University, Bangladesh
}

\begin{abstract}
Correspondence: Muhammad Torequl Islam, Department of Pharmacy, Life Science Faculty, Bangabandhu Sheikh Mujibur Rahman Science and Technology University, Gopalganj 8100, Bangladesh, Tel +8801793726407, Email dmt.islam@bsmrstu.edu.bd
\end{abstract}

Received: April 23, 2020 | Published: August 07, 2020

Keywords: SARS-CoV-2; covid-19; diatery contents; vitamins

Abbreviations: TGF- $\beta$, tumor growth factor beta; IL, interleukin; ROS, reactive oxygen species; NK, natural killer; GCSF; granulocyte-colony stimulating factor; EBOV, Ebola virus; IAV, influenza A virus; ARBs, angiotensin receptor blockers

\section{Letter to Editor}

The coronavirus disease 2019 (Covid-19) has been gained much attention as it has been able to negatively affect from all areas worldwide. Every day it is calming thousands of lives across the world. ${ }^{1}$ Although, we have taken many measures, but the number of infected cases is grossly increasing daily still. Unfortunately, there is no specific treatment(s) for it. To date 165 vaccine candidates from many countries around the world have been undergoing in various phases of clinical trials. Among them, 26 vaccine candidates are under human trials. The Oxford University's vaccine has been stepped forward than the others, showing a hope to people worldwide. However, still it's a long way to go. On the other hand, the discovery of vaccines is also delaying the conventional drug therapy approaches. Thus, raising the question- In this current situation, what actually we do? A number of protective measures such as isolation, quarantine, social distancing, avoidance of large gatherings, avoidance close contact with people suffering from acute respiratory infections, frequent hand-washing, especially after direct contact with ill people or their environment, and avoiding unprotected contact with farm or wild animals have been suggested by the experts around the world. ${ }^{2-4}$ Respiratory and/ or circulatory supports along with some other antiviral drug therapies have been also reported. However, along with the protection and treatment strategies, dietary supplements and nutritional behavior may help us to manage Covid-19. This writing focuses on the effects of dietary contents, especially the diets containing vitamins on the management of Covid-19.

Nutritional and pathogenic stress interact with the physiological malfunctions in animals, ${ }^{5}$ as these can lead to hepatic, ${ }^{6}$ cardiovascular, ${ }^{7}$ neurological ${ }^{8}$ and many other diseases. Diets that are rich in polysaccharides (e.g., cereals, onion, garlic, ginger, algae) along with protein and fat derived from fish and soy are necessary for healthy livelihood. Natural antioxidants such as quercetin and alliin are evident to increase the presence of Firmicutes genera, while decrease in Bacteroidetes and Proteobacteria in the intestine, therefore, reduces the incidence of inflammatory bowel disease. ${ }^{9}$ Diets containing high amount of unsaturated fatty acids (e.g., linolenic, linoleic, eicosapetaenoic, docoshexaenoic, and arachidonic acids), omega-3 and omega- 6 fatty acids found in large quatities in fish and nuts, as well as low glycemic index carbohydrates, are evident to promote healthy metabolic profiles in human. ${ }^{10}$ Generally, foods containing high carbohydrate and fat, and low fiber have profound effects on the regulation of our immune system. ${ }^{11}$

Diets modified seasonally or by biogeographic, demographic or cultural shifts may contain lower amounts of essential nutrients and can differentially influence mitochondrial bioenergetics, retrograde signalling to the nuclear genome, and anterograde signalling to mitochondria, alter the frequencies of mtDNA haplotypes and affect the health of human and other animals. ${ }^{12}$ Food supplements may be a potential source to manage the Covid-19. In a study, feed additives such as phosphoric acid, citric acid, or fumaric acid were found to reduce porcine delta coronavirus $(\mathrm{CoV})$ in infected pigs. ${ }^{13}$ In another study, chick embryo tracheal organ cultures showed increased resistance to infection by a $\mathrm{CoV}$ after exposure to ascorbate (also called vitamin C. ${ }^{14}$ Generally, vitamins act as modulators of immunological functions, including activation, proliferation and cytokine production by $\mathrm{T}$ cells.

Intestinal dendritic cells use vitamin A to produce retinoic acid, which activates the mucosal immune system, and displays a number of other important functions, including production of chemokines, adhesion molecules, and specific cytokines (e.g., tumor growth factor beta (TGF- $\beta$ ) and interleukin (IL)-17), promotes the differentiation and activation of intestinal CD4+ Th17 effector cells, CD4+ FoxP3+ Treg cells, and IgA-producing plasma cells. ${ }^{15,16}$ The vitamins of B group also play important roles for immune system homeostasis and functions. ${ }^{17}$ Thiamine (vitamin $\mathrm{B}_{1}$ ) promotes accumulation of naïve $\mathrm{B}$ cells in intestinal Peyer patches and induces IgA production in the intestinal mucosa. ${ }^{17}$ It also regulates leukocyte activation, 
proliferation, and production of pro-inflammatory cytokines upon antigenic stimulation. ${ }^{18}$ On the other hand, riboflavin (vitamin $\mathrm{B}_{2}$ ) deficiency has been evident to impart a negative effect on macrophage cell lines, decrease in cell adhesion, viability, production of reactive oxygen species (ROS), and phagocytic activity. ${ }^{19}$ Niacin (vitamin $\mathrm{B}_{3}$ ) has anti-inflammatory property. ${ }^{20}$ Pyridoxal phosphate (vitamin $\mathrm{B}_{6}$ ), a coenzyme necessary for carbohydrate, lipid, heme and amino acid metabolism. It has anti-inflammatory and antioxidant effects. ${ }^{21}$ In a study, a diet without $\mathrm{B}_{6}$ showed a decreased $\mathrm{T}$ cell proliferation, differentiation, and IL-2 production, with an enhanced IL-4 secretion after mitogenic stimulation in mice. ${ }^{22}$ Biotin (vitamin $\mathrm{B}_{7}$ ) deficiency inhibit the proliferation and maturation of $\mathrm{B}$ and $\mathrm{T}$ lymphocytes decreasing antibody production by B cells and cytotoxic activity of natural killer (NK) and T cells. ${ }^{23}$ Folate (vitamin $\mathrm{B}_{9}$ ) interacts with vitamins $\mathrm{B}_{6}$ and $\mathrm{B}_{12}$, as well as homocysteine, in trans-methylation metabolic pathways that regulate various cell functions, including the response to oxidative stress. ${ }^{24}$ It also regulates pro-inflammatory and effector functions in leukocytes. On the other hand, cobalamin (vitamin $\mathrm{B}_{12}$ ) deficiency has been found to increase IL-6 production by peripheral blood mononuclear cells in Alzheimer disease patients. ${ }^{25}$

Vitamin $\mathrm{C}$ is evident to manage common cold in experimental animals. ${ }^{26}$ A deficiency of this vitamin causes immune system dysfunction and increased susceptibility to infections. It promotes chemotaxis, phagocytic activity, and bacterial killing of neutrophils and macrophages, as well as lymphocyte proliferation and differentiation, production of antibodies by $\mathrm{B}$ cells and cytotoxic activity of NK cells..$^{27,28}$ On the other hand, vitamin D regulates a number of important functions within the host. It maintains epithelial homeostasis and absorption of phosphate and calcium in the intestine, and helps in the development and proper functioning of the immune system. ${ }^{29}$ Vitamin E enhances lymphocyte proliferation, NK celldependent cytotoxicity, Th1 responses, antibody production, and secretion of cytokines (e.g., IL-1 $\beta$ and IL-2) and also enhances the antibacterial activity of neutrophils and inhibits oxidative damage and apoptosis in macrophages. ${ }^{30,31}$

Many $\mathrm{CoV}$ proteins are modified by post-translational modifications, including glycosylation and palmitoylation of the spike and envelope protein, $\mathrm{N}$ - or $\mathrm{O}$-linked glycosylation of the membrane protein, phosphorylation and ADP-ribosylation of the nucleocapsid protein, and other post-translational modifications on non-structural and accessory proteins. ${ }^{32} \mathrm{CoV}$ infection triggers cellular stress responses in infected host cells, which inhibits the global protein synthesis to reduce the amount of unfolded proteins inside the endoplasmic reticulum lumen, results in overcoming the protein translation shutoff to ensure viral protein production. ${ }^{33}$ The SARS-CoV-2 has been evident to increase the levels of plasma pro-inflammatory cytokines, including IL2, IL7, IL10, granulocytecolony stimulating factor (GCSF), interferon gamma-induced protein 10 (IP10), monocyte chemoattractant protein 1 (MCP1), macrophage inflammatory protein (MIP) $1 \alpha$, and tumor necrosis factor alpha $(\mathrm{TNF} \alpha) \cdot{ }^{34}$ Therefore, the vitamins, minerals and other nutrients present in our diets having antioxidant and anti-inflammatory capacity may encounter the oxidative stress and inflammation induced by SARS$\mathrm{CoV}-2$ in host cells. In a study, ginkgolic acids have been reported to treat acute infections caused by a number of viruses, including CoVs, Ebola virus (EBOV), Zika virus (ZIKV), influenza A virus (IAV), measles, Jerpes simplex viruses (e.g., HSV-1, HSV-2) and varicellazoster. ${ }^{35}$ Several herbs and traditional medicines (e.g., ginseng, gingko biloba), flavonoids, and other nutrients (magnesium, zinc, carnitine, omega-3 fatty acids) as well as vitamins (e.g., $\left.\mathrm{B}_{2}, \mathrm{~B}_{3}, \mathrm{~B}_{6}, \mathrm{~B}_{9}, \mathrm{C}, \mathrm{D}, \mathrm{E}\right)$ have been reported to treat brain injury. ${ }^{36}$ Wheeler et al. ${ }^{37}$ demonstrated that healthy microglia are required for the protection against lethal $\mathrm{CoV}$ encephalitis in mice.

The human receptor angiotensin converting enzyme 2 receptor (ACE2R), a transmembrane protein, serves as the main entry point into cells for SARS-CoV-2. ${ }^{38}$ Therefore, ACE inhibitors and angiotensin receptor blockers (ARBs) that are used to treat high blood pressure may upregulate ACE2R expression, and may affect the severity of coronavirus infection. ${ }^{38,39}$ ACE2R overexpression reduces oxidative stress and cyclooxygenase (COX)-mediated inflammation, ${ }^{40}$ which might be helpful for SARS-CoV-2's existence and replication in the host cells. The compensatory activity of ACE2 is impaired by the disintegrin and metalloprotease 17 (ADAM17), and lack of ACE2 is associated with oxidative stress. Alpha-lipoic acid, a vitamin-like chemical and an antioxidant was seen to downregulate ADAM17 and preserve ACE2 compensatory activity in Neuro2A cells. ${ }^{41}$ Calcitriol (active form of vitamin D) modulated the expression of ACE1 and ACE2 in lipopolysaccharide-induced lung injury Wistar rats. ${ }^{42}$ In another study, this vitamin also attenuated angiotensin II-induced nitrogen oxides (NOx) activation and ROS production. ${ }^{43}$ However, high glucose diet can activate ACE2R in human. ${ }^{44}$

\section{Conclusion}

In summary, diets containing natural antioxidants and vitamins may be helpful to fight against viral infections, including the SARSCoV-2. Hopefully, to date a number of clinical trials have been undergoing on vitamins, including vitamin A (e.g., retinol palmitate, derivative of vitamin $\mathrm{A}$ ), C and D. Vitamins can stimulate and strengthen our immune system; help us to protect against infectious diseases, including viral infection. Moreover, by this way sufficiently produced antibody may act against SARS-CoV-2.

\section{Acknowledgments}

None.

\section{Conflicts of interest}

Author declares that there are no conflicts of interest.

\section{Funding}

None.

\section{References}

1. World Health Organization (WHO). Clinical management of severe acute respiratory infection when novel coronavirus $(\mathrm{nCoV})$ infection is suspected. Swizerlan, Geneva: World Health Organization; 2020.

2. Du Z, Wang L, Cauchemez S, et al. Risk for transportation of 2019 novel coronavirus disease fromWuhan to other cities in China. Emerg Infect Dis. 2020;26(5).

3. McCloskey B, Heymann DL. SARS to novel coronavirus: old lessons and new lessons. Epidemiol Infect. 2020;148:e22.

4. Wilder-Smith A, Freedman DO. Isolation, quarantine, social distancing and community containment: pivotal role for old-style public health measures in the novel coronavirus $(2019-\mathrm{nCoV})$ outbreak. $J$ Travel Med. 2020;27(2):taaa020. 
5. Rutter L, Carrillo-Tripp J, Bonning BC, et al. Transcriptomic responses to diet quality and viral infection in Apis mellifera. BMC Genomics. 2019;20(1):412.

6. Weiskirchen S, Weiper K, Tolba RH, et al. All You Can Feed: Some Comments on Production of Mouse Diets Used in Biomedical Research with Special Emphasis on Non-Alcoholic Fatty Liver Disease Research. Nutrients. 2020;12(1):163.

7. Naude CE, Schoonees A, Senekal M, et al. Low Carbohydrate versus Isoenergetic Balanced Diets for Reducing Weight and Cardiovascular Risk: A Systematic Review and Meta-Analysis. PLoS One. 2014;9(7):e100652.

8. Estrada JA, Contreras I. Nutritional Modulation of Immune and Central Nervous System Homeostasis: The Role of Diet in Development of Neuroinflammation and Neurological Disease. Nutrients. 2019;11(5):1076.

9. Yu J, Guo H, Xie J, et al. The Alternate Consumption of Quercetin and Alliin in the Traditional Asian Diet Reshaped Microbiota and Altered Gene Expression of Colonic Epithelial Cells in Rats. J Food Sci. 2019;84(3):678-686.

10. Oliviero F, Spinella P, Fiocco U, et al. How the Mediterranean diet and some of its components modulate inflammatory pathways in arthritis. Swiss Med Wkly. 2015;145:w14190.

11. Martinez KB, Leone V, Chang EB. Western diets, gut dysbiosis, and metabolic diseases: Are they linked? Gut Microbes. 2017;8(2):130 142

12. Aw WC, Towarnicki SG, Melvin RG, et al. Genotype to phenotype: Diet-by-mitochondrial DNA haplotype interactions drive metabolic flexibility and organismal fitness. PLoS Genet. 2018;14(11):e1007735.

13. Kottingim KM, Verma H, Urriola P, et al. Feed additives decrease survival of delta coronavirus in nursery pig diets. Porcine Health Manag. 2017;3:5.

14. Atherton JG, Kratzing CC, Fisher A. The effect of ascorbic acid on infection of chick-embryo ciliated tracheal organ cultures by coronavirus. Arch Virol. 1978;56(3):195-199.

15. Mora JR, Iwata M, Eksteen B, et al. Generation of gut-homing IgA-secreting B cells by intestinal dendritic cells. Science. 2006;314(5802):1157-1160.

16. Coombes JL, Siddiqui KR, Arancibia-Carcamo CV, et al. A functionally specialized population of mucosal CD103+ DCs induces Foxp3+ regulatory $\mathrm{T}$ cells via a TGF-beta and retinoic acid-dependent mechanism. J Exp Med. 2007;204(8):1757-1764.

17. Kunisawa J, Sugiura Y, Wake T, et al. Mode of Bioenergetic Metabolism during B Cell Differentiation in the Intestine Determines the Distinct Requirement for Vitamin B1. Cell Rep. 2015;13(1):122-131.

18. Bozic I, Savic D, Laketa D, et al. Benfotiamine attenuates inflammatory response in LPS stimulated BV-2 microglia. PLoS One. 2015;10(2):e0118372.

19. Mazur-Bialy AI, Buchala B, Plytycz B. Riboflavin deprivation inhibits macrophage viability and activity-a study on the RAW 264.7 cell line. Br J Nutr. 2013;110(3):509-514.

20. Singh N, Gurav A, Sivaprakasam S, et al. Activation of Gpr109a receptor for niacin and the commensal metabolite butyrate, suppresses colonic inflammation and carcinogenesis. Immunity. 2014;40(1):128139

21. Zhang P, Tsuchiya K, Kinoshita T, et al. Vitamin B6 Prevents IL-1 $\beta$ Protein Production by Inhibiting NLRP3 Inflammasome Activation. $J$ Biol Chem. 2016;291:24517-24527.
22. Qian B, Shen S, Zhang J, et al. Effects of Vitamin B6 Deficiency on the Composition and Functional Potential of T Cell Populations. J Immunol Res. 2017;2017:2197975.

23. Kuroishi T. Regulation of immunological and inflammatory functions by biotin. Can J Physiol Pharmacol. 2015;93(130:1091-1096.

24. Kruman II, Kumaravel TS, Lohani A, et al. Folic acid deficiency and homocysteine impair DNA repair in hippocampal neurons and sensitize them to amyloid toxicity in experimental models of Alzheimer's disease. J Neurosci. 2020;22(5):1752-1762.

25. Watanabe $\mathrm{S}$, Ide $\mathrm{N}$, Ogawara $\mathrm{H}$, et al. High percentage of regulatory $\mathrm{T}$ cells before and after vitamin B12 treatment in patients with pernicious anemia. Acta Haematol. 2015;133:83-88.

26. Yakoot M, Salem A. Efficacy and safety of a multiherbal formula with vitamin $\mathrm{C}$ and zinc (Immumax) in the management of the common cold. Int J Gen Med. 2011;4:45-51.

27. Hemilä H. Vitamin C and Infections. Nutrients. 2017;9(4):339.

28. Van Gorkom GNY, Klein Wolterink RGJ, Van Elssen CHMJ. et al. Influence of Vitamin C on Lymphocytes: An Overview. Antioxidants. 2018;7(3):41

29. Dimitrov V, White JH. Vitamin D signaling in intestinal innate immunity and homeostasis. Mol Cell Endocrinol. 2017;453:68-78.

30. Chen W, Bichara DA, Suhardi J, et al. Effects of vitamin E-diffused highly cross-linked UHMWPE particles on inflammation, apoptosis and immune response against S. aureus. Biomaterials. 2017;143:46-56.

31. Lee GY, Han SN. The Role of Vitamin E in Immunity. Nutrients. 2018;10(11):1614.

32. Fung TS, Liu DX. Post-translational modifications of coronavirus proteins: roles and function. Future Virol. 2018;13(6):405-430

33. Fung TS, Liao Y, Liu DX. Regulation of Stress Responses and Translational Control by Coronavirus. Viruses. 2016;8(7):184.

34. Huang C, Wang Y, Li X, et al. Clinical features of patients infected with 2019 novel coronavirus in Wuhan, China. Lancet 2020;395(10223):497-506

35. Borenstein R, Hanson BA, Markosyan RM, et al. Ginkgolic acid inhibits fusion of enveloped viruses. Sci Rep. 2020;10(1):4746

36. Haar CV, Peterson TC, Martens KM, et al. Vitamins and Nutrients as Primary Treatments in Experimental Brain Injury: Clinical Implications for Nutraceutical Therapies. Brain Res. 2016;1640(Pt A):114-129.

37. Wheeler DL, Sariol A, Meyerholz DK, et al. Microglia are required for protection against lethal coronavirus encephalitis in mice. J Clin Invest. 2018;128(3):931-943.

38. Walls AC, Park YJ, Tortorici MA, et al. Structure, function and antigenicity of the SARS-CoV-2 spike glycoprotein. Cell. 2020;181(2):281-292.e6.

39. Diaz JH. Hypothesis: angiotensin-converting enzyme inhibitors and angiotensin receptor blockers may increase the risk of severe COVID-19. J Travel Med. 2020;27(3).

40. Sriramula S, Xia H, Xu P, et al. Brain-targeted ACE2 overexpression attenuates neurogenic hypertension by inhibiting COX mediated inflammation. Hypertension. 2015;65(3):577-586.

41. de Queiroz TM, Xia H, Filipeanu CM. et al. $\alpha$-Lipoic acid reduces neurogenic hypertension by blunting oxidative stress-mediated increase in ADAM17. Am J Physiol Heart Circ Physiol. 2015;309(5):H926H934. 
42. Xu J, Yang J, Chen J, et al. Vitamin D alleviates lipopolysaccharideinduced acute lung injury via regulation of the renin-angiotensin system. Mol Med Rep. 2017;16(5):7432-7438.

43. Cui C, Xu P, Li G, et al. Vitamin D receptor activation regulates microglia polarization and oxidative stress in spontaneously hypertensive rats and angiotensin II-exposed microglial cells: Role of renin-angiotensin system. Redox Biol. 2019;26:101295.
44. Härdtner C, Mörke C, Walther R, et al. High glucose activates the alternative ACE2/Ang-(1-7)/Mas and APN/Ang IV/IRAP RAS axes in pancreatic $\beta$-cells. Int J Mol Med. 2013;32(4):795-804. 